\title{
Numerical simulations of kinematic dynamo action
}

\author{
V. Archontis ${ }^{1}$, S. B. F. Dorch ${ }^{2,3}$, and $\AA$. Nordlund ${ }^{3}$ \\ 1 Instituto de Astrofisica de Canarias, Via Lactea s/n, 38200 La Laguna, Spain \\ 2 The Institute for Solar Physics of the Royal Swedish Academy of Sciences, SCFAB, 10691 Stockholm, Sweden \\ 3 The Niels Bohr Institute for Astronomy, Physics and Geophysics, Juliane Maries Vej 30, 2100 Copenhagen Ø, Denmark
}

Received 2 May 2002 / Accepted 21 October 2002

\begin{abstract}
Numerical simulations of kinematic dynamo action in steady and three-dimensional $A B C$ flows are presented with special focus on the difference in growth rates between cases with single and multiple periods of the prescribed velocity field. It is found that the difference in growth rate (apart from a trivial factor stemming from a scaling of the rate of strain with the wavenumber of the velocity field) is due to differences in the recycling of the weakest part of the magnetic field. The single wavelength classical $A B C$-flow experiments impose stronger symmetry requirements, which results in a suppression of the growth rate. The experiments with larger wave number achieve growth rates that are more compatible with the turn-over time scale by breaking the symmetry of the resulting dynamo-generated magnetic field. Differences in topology in cases with and without stagnation points in the imposed velocity field are also investigated, and it is found that the cigar-like structures that develop in the classical $A=B=C$ dynamos are replaced by ribbon structures in cases where the flow is without stagnation points.
\end{abstract}

Key words. diffusion - magnetic fields - magnetohydrodynamics (MHD) - turbulence - plasmas

\section{Introduction}

Dynamo theory aims at explaining how magnetic fields can be generated and sustained by fluid motions in the presence of dissipation. Studies of dynamo action in astrophysical objects are usually connected with the question of the existence of fast dynamos (Vainshtein \& Zeldovich 1972). The fast dynamo problem refers to the generation of magnetic fields in the limit of infinite magnetic Reynolds number $R e_{\mathrm{m}}$, and has been extensively reviewed by e.g. Childress \& Gilbert (1995).

In the simplest formulation of the kinematic dynamo problem, an initially weak magnetic field is amplified due to the motions of a prescribed, stationary velocity field. Flows that have chaotic transport properties are prime candidates for fast dynamos. They have the ability to amplify the magnetic field exponentially by stretching the magnetic field lines. One such class of flows that is often invoked in fast dynamos studies is the $A B C$ flows (named after Arnold, Beltrami \& Childress). A first detailed report of the kinematic dynamo action of $A B C$ flows may be found in Dombre et al. (1986), who examined especially the internal symmetries of the velocity field. There is, however, no guarantee that such symmetries are preserved in the eigenmodes of the magnetic field. Some discussion of possible symmetry breaking in the eigenmodes may be found in Galloway et al. (1984) and in Galanti et al. (1992). They found no evidence of symmetry breaking when the magnetic

Send offprint requests to: $\mathrm{V}$.D. Archontis, e-mail: vasilis@ll.iac.es
Reynolds number is limited to very low values, although symmetries are broken for higher values of the magnetic Reynolds number. The presence of symmetry breaking was also shown by Galloway et al. (1984) when the wavenumber of the periodic $A B C$ flow is equal to 3 and 4 . The conclusion was that the physical reason for the symmetry breaking is unclear and requires further investigation.

Dynamo action in $A B C$-flows has also been studied to determine the growth rate of the magnetic field for different values of the magnetic Reynolds number (Arnold et al. 1983; Galloway et al. 1984; Lau \& Finn 1993). Results for the case with unit wavenumber, for magnetic Reynolds number up to $R e_{\mathrm{m}}=1600$ (Dorch 2000) showed that the growth rate saturates at a value close to 0.07. Galanti et al. (1992) found that doubling the wavenumber of the flow leads to a sharp increase of the growth rate, while for larger wavenumbers the growth rate scales with the strain rate of the flow (i.e., in proportion to the wave number).

In the current paper we address some of the issues mentioned above by means of numerical kinematic MHD simulations. The dynamics of the magnetic structures in physical space is examined in connection with the amplification mechanism which drives the dynamo action in $A B C$ flows. These flows become hydrodynamically unstable at low kinetic Reynolds number, Re (Podvigina \& Pouquet 1994). We briefly discuss the dependence of the growth rate on $R e_{\mathrm{m}}$ but defer a detailed discussion of this topic to a forthcoming paper, and concentrate on the dynamo properties of laminar $A B C$ flows in 
the current paper. In particular, we investigate, the physical reason that causes the increase of the growth rate in an $A B C$ flow for wavenumbers larger than unity. To achieve this we follow the motion of magnetic field lines in time and demonstrate that the transportation of the weak part of the field is a key factor of the dynamo process.

The numerical aspects of the simulations are described in the following section. The growth rates produced by fast kinematic dynamo action of $A B C$ flows are presented in Sect. 3. Visualizations of the magnetic structures and field lines in physical space are shown in Sect. 4. Dynamo action produced by flows without stagnation points is studied in Sect. 5 . Section 6 contains the overall conclusions of the numerical simulations presented here.

\section{The simulation}

Our aim is to study kinematic dynamo action in $A B C$ flows. The time evolution of $\boldsymbol{B}$ is governed by the (dimensionless) induction equation:

$\frac{\partial \boldsymbol{B}}{\partial t}=\nabla \times(\boldsymbol{u} \times \boldsymbol{B})+\frac{1}{R e_{\mathrm{m}}} \nabla^{2} \boldsymbol{B}$

$\nabla \cdot \boldsymbol{B}=\nabla \cdot \boldsymbol{u}=0$.

The velocity field is chosen to have the form of an $A B C$ flow:

$$
\begin{aligned}
\boldsymbol{u}_{A B C}= & A(0, \sin k x, \cos k x)+B(\cos k y, 0, \sin k y) \\
& +C(\sin k z, \cos k z, 0),
\end{aligned}
$$

where $k$ is the wavenumber of the flow. The special case with parameters $A=B=C=k=1$ is referred to as the "normal" $A B C$ flow dynamo. In the normal case there are two windows of dynamo action (where the growth rates are positive): $A$ closed one in the interval $R e_{\mathrm{m}}=8.9-17.5$, and an open one for magnetic Reynolds numbers above 27 (Galloway et al. 1984).

Equation (1) is solved numerically on a staggered mesh using array valued functions to evaluate the space and time derivatives. A third-order predictor-corrector method (Hyman 1979) is used for the time-stepping. The applied numerical scheme (by Galsgaard, Nordlund and others, see Galsgaard \& Nordlund 1997; Nordlund et al. 1992) ensures Eq. (2). Periodicity of $2 \pi$ is assumed over a three-dimensional computational domain. The initial magnetic field is a random perturbation with an amplitude of $10^{-5}$.

\section{Growth rates}

Previous numerical simulations (e.g. Arnold et al. 1983; Galloway et al. 1984; Galanti et al. 1992; Dorch 2000) have shown that the normal $(A=B=C=k=1) A B C$ flow very likely acts as a fast dynamo with a growth rate close to 0.077 (corresponding to a time-scale of $\approx 0.25$ turn-over times). When the wavenumber $k$ is larger than unity (where $k=1$ corresponds to the largest scale of $2 \pi$ in the computational domain) the growth rate scales approximately with the rate of strain. These results are confirmed in our simulations and we continue the study of the growth rate of the magnetic field for higher values of $R e_{\mathrm{m}}$.
We first discuss the case with $A=B=C=1$ and $k=2$ before turning our attention to higher wavenumbers: As shown in Fig. 1 the magnetic field is amplified exponentially without oscillations. Initially, several modes may be present, associated with oscillatory growth of the magnetic field (as in the case of a normal $A B C$ flow, Dorch 2000), but eventually the mode with the highest growth rate is the only one that remains in the solution (i.e. the dominant mode).

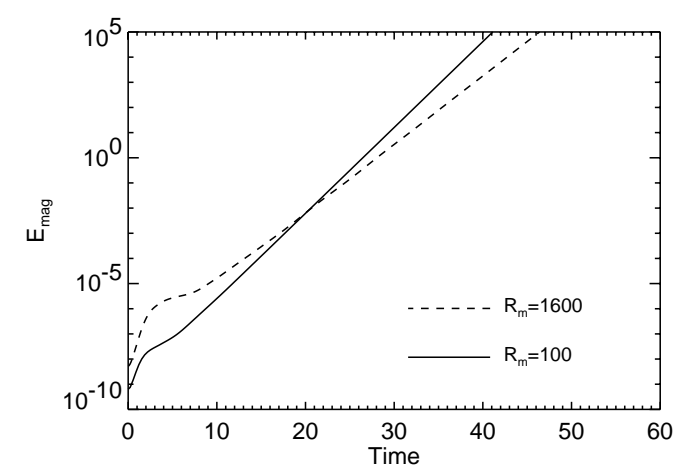

Fig. 1. Temporal evolution of the magnetic energy for the kinematic $A B C$ dynamo with $A=B=C=1$ and $k=2$ for $R e_{\mathrm{m}}=100$ and $R e_{\mathrm{m}}=1600$.

For low $R e_{\mathrm{m}}$ the growth rate increases with $R e_{\mathrm{m}}$ and reaches its maximum value at $R e_{\mathrm{m}}=12$. This value of the magnetic Reynolds number belongs to the first dynamo window for the normal $A B C$ flow dynamo, and corresponds to a maximum growth rate of the magnetic field in this window (Galanti et al. 1992). With $k=2$ the lowest $R e_{\mathrm{m}}$ possible for dynamo action is found to be approximately equal to 2 . For large $R e_{\mathrm{m}}$ the growth rate decreases slightly (in contrast to the low $R e_{\mathrm{m}}$ results of Galanti et al. 1992) but always remains positive and bounded away from zero (Fig. 2). The largest value of $R e_{\mathrm{m}}$ considered here is $R e_{\mathrm{m}}=1600$ and the corresponding numerical resolution is $128^{3}$. In the high $R e_{\mathrm{m}}$ regime the growth rate saturates at a value close to 0.3 .

The evidence indicates that the $A B C$ flow with $k=2$ is a fast dynamo, since the growth rate seems to saturate at a nonzero value for high $R e_{\mathrm{m}}$. The reason for the enhancement of the growth rate when $k=2$ is discussed in the following section.

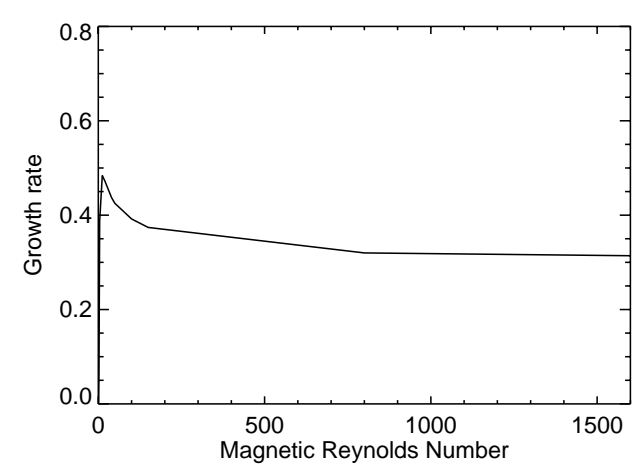

Fig. 2. Growth rate of the magnetic energy (normalized by the rate of strain) versus the magnetic Reynolds number for the kinematic $A B C$ dynamo with $A=B=C=1$ and $k=2$. 

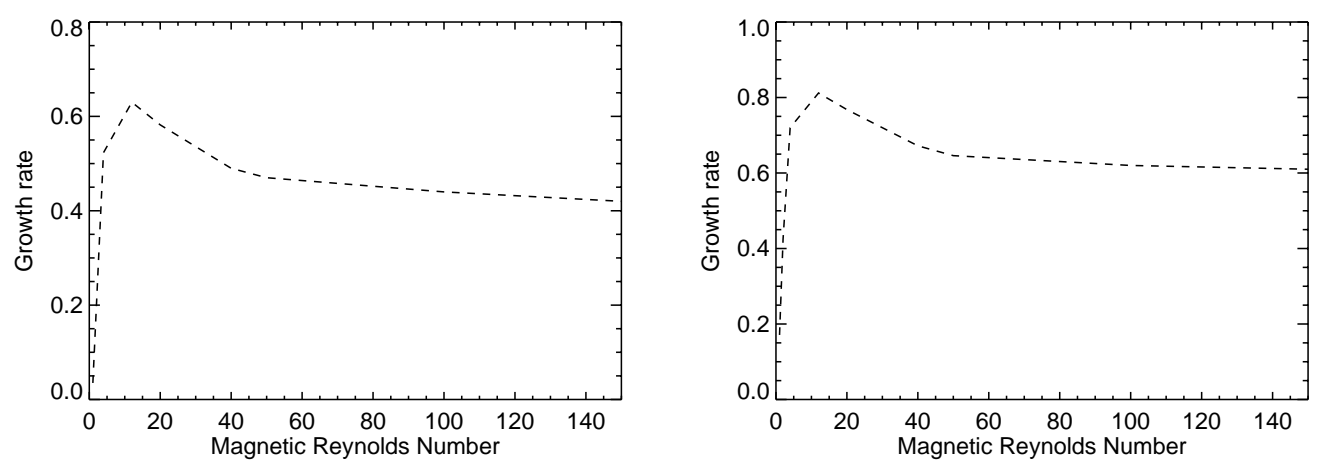

Fig. 3. Two panels showing the growth rate of the magnetic energy normalized by the rate of strain for the kinematic $A B C$ dynamo with $k=3$ (left panel) and $k=4$ (right panel).
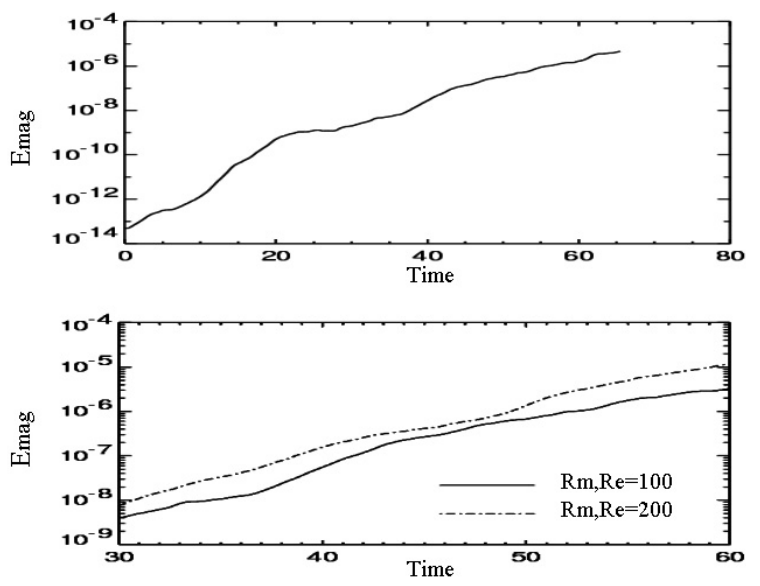

Fig. 4. Temporal evolution of the magnetic energy for the normal $A B C$ flow with $R e_{\mathrm{m}}=R e=100$ (top). Comparison with $R e_{\mathrm{m}}=R e=200$ over a long stretch of the kinematic regime (bottom).

The results for the growth rate of the magnetic energy at different values of magnetic Reynolds number when $k=3$ and $k=4$ are shown in Fig. 3. A comparison of these results with the $k=2$ case (Fig. 2) shows that the growth rate of the magnetic field increases with increasing wave number, in approximate proportionality to $k$ for $k \geq 2$, scaling approximately as $0.15 \times k$, while the growth rate is unproportionally small $(\approx 0.07)$ for $k=1$.

The normal $A B C$ flow loses its stability at moderate values of $R e$ and becomes increasingly more complex as $R e$ increases (Podvigina \& Pouquet 1994). However, as we discuss in more detail in a forthcoming paper, the growth rate is not significantly different in the nonlinear flows that develop at high $R e$, provided that the forcing is controlled so as to keep the turnover times approximately the same. An experiment with $R e_{\mathrm{m}}=R e=100$ at $40^{3}$ resolution shows that the magnetic field is amplified exponentially with an average growth rate close to 0.064 (Fig. 4, top). Doubling the values of Reynolds numbers and of the resolution, the temporal evolution of the magnetic energy is examined and it is found that, apart from a transient increase of the magnetic energy as the structures shrink in size, the growth rate is very similar (Fig. 4, bottom). This result indicates that the growth rate is mainly determined by the large scale properties of the flow (in particular the turn over time), and we expect that the only important change in the magnetic field structure with increasing Reynolds number is the development of more fine structure.

\section{Magnetic structures}

An experiment with $R e_{\mathrm{m}}=100$ and a numerical resolution of $64^{3}$ was performed in order to study the dynamics of the magnetic structures in physical space when the wavenumber $k$ of the flow is equal to 2 . The computational domain then contains eight periodic cells, where each one (initially) reflects the geometrical properties of a standard $A B C$ flow. The magnetic field evolves according to the induction equation Eq. (1) and flux "cigars" are formed at the so-called $\alpha$-type stagnation points (Dombre et al. 1986). These are points (stable manifolds of the flow) with converging streamlines in the plane of the stagnation point and the field is rapidly advected to these regions (Fig. 5). The flux cigars are aligned along the axis of divergence through the $\alpha$-type stagnation points.

At the $\beta$-type stagnation points (unstable manifolds of the flow) the field is weaker, and takes the form of flux sheets on both sides of the plane of divergence. The flux sheets are formed because of the converging flow along the axis of divergence from the $\alpha$-type stagnation points. The flux cigars thus appear to be connected by flux sheets through heteroclinic stream lines.

When the symmetry breaking appears, and the fastest growing eigenmode takes over, the topology of the magnetic field changes. The fastest growing mode does not follow the symmetric configuration of a standard $A B C$ flow - the strong magnetic field is still elongated into flux "cigars" but their size and field strength vary from cell to cell (Fig. 6).

The rate of growth (and decay) of individual flux cigars is different, rather than being uniform as in the normal $k=1$ case. The magnetic field for the latter case is recycled inside its cell because of the exact symmetry. The time evolution of the magnetic energy follows a periodic (oscillatory) behavior which is associated with the conservation of symmetry in the fastest growing eigenmode (Dorch 2000) in contrast to the dominant mode for the $k=2$ case which is a symmetry breaking mode (Galloway et al. 1984). Visualization of magnetic field lines shows that they are not "trapped" in each individual cell but there is always a "loose" weak field which is transported from 


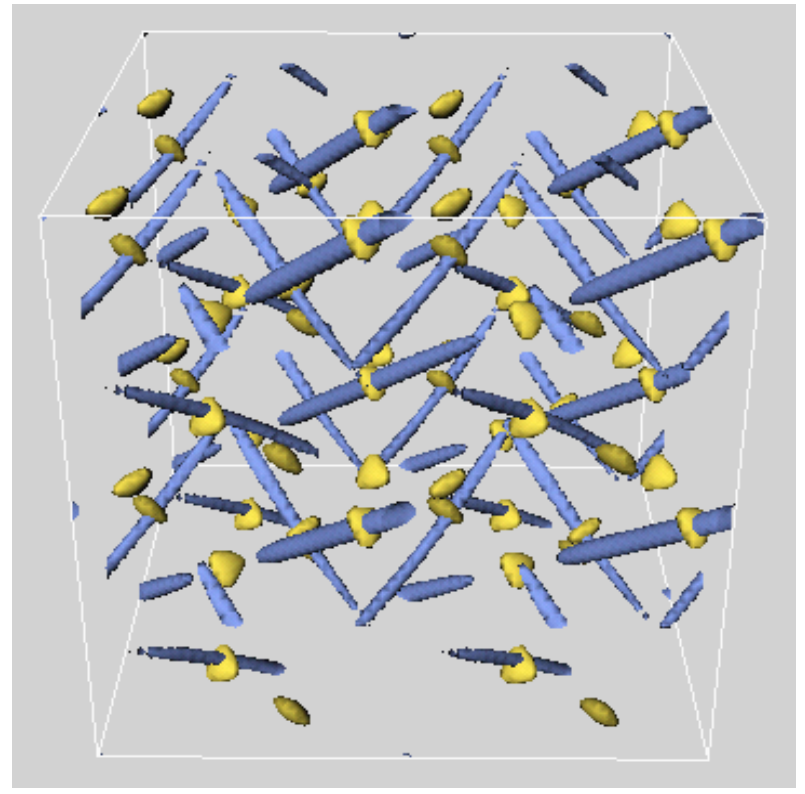

Fig. 5. Magnetic field strength isosurfaces (dark) and stagnation points of the flow (light). The magnetic field is concentrated along flux cigars centered at the $\alpha$-type stagnation points of the flow. The stagnation points with no flux cigars centered at them are the $\beta$-type points. In each cell three cigars form a triangle around a $\beta$-type stagnation point and another one is pointing towards it. The magnetic field strength of the isosurfaces is $70 \%$ of the peak value in the snapshot.

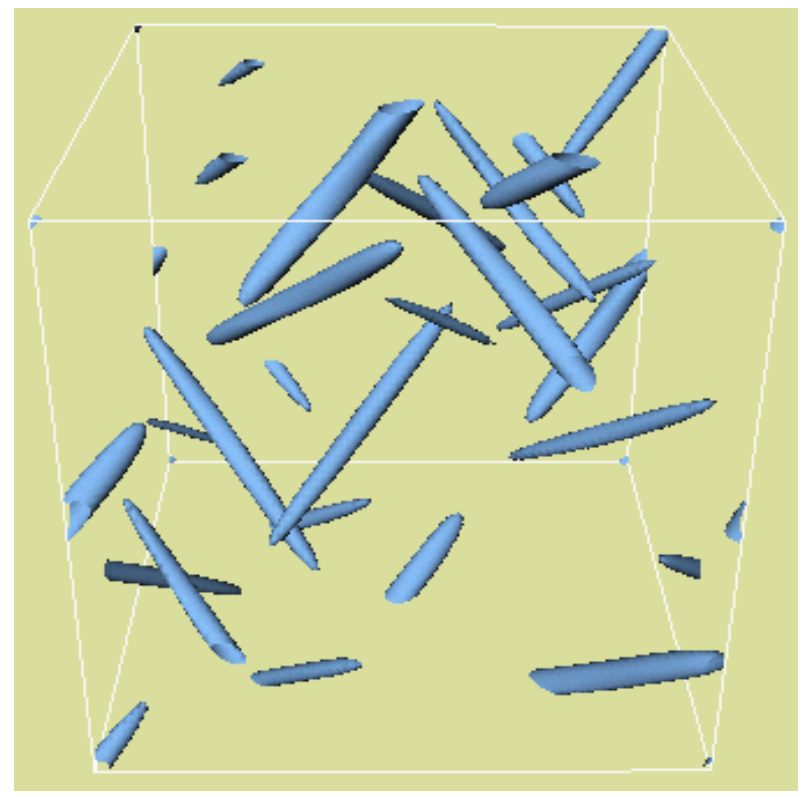

Fig. 6. A snapshot showing the structure of the magnetic field when the fastest growing mode appears and the symmetry is not conserved. Each cell consists of flux cigars which have different size and different field strength.

cell to cell and is stretched up against the local flux cigars that are passed on the way (Fig. 7). In general, the increase of the magnetic energy is associated with the stretching, twisting and folding of the magnetic field lines against the local flux cigars in the $\beta$-type planes. The field lines coming from a flux cigar

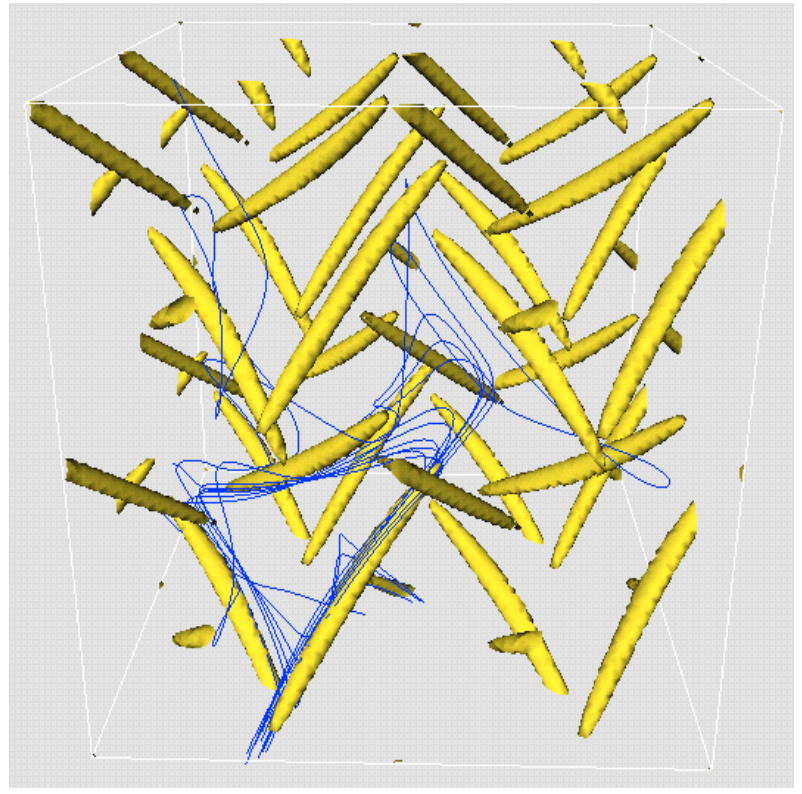

Fig. 7. Visualization of magnetic field lines and flux cigars when the dominant mode starts to take over. The stretching of the field lines at the corner of a triangle at the bottom-left cell is obvious. These field lines get pressed up against another cigar in a neighboring cell (up-right).

are connected through the weak field to the cigars that form the triangles around the $\beta$-type stagnation points. The field lines at the corners of the triangle are stretched and added to other flux cigars of neighboring cells through the diverging stream lines. The flux sheets that are formed on both sides of the $\beta$ type plane are also folded constructively. The $\beta$-type plane is actually a plane of discontinuity where the magnetic field lines change direction and are released to move back into the general flow (Fig. 8).

\section{Flows without stagnation points}

In view of the role played by the stagnation points in the normal $A B C$ flow dynamo, it is natural to ask whether they are a necessary ingredient for fast dynamo action. When the parameters $A$, $B$ and $C$ are varied it is possible for the stagnation points to be created or destroyed (Dombre et at. 1986). When $A=5, B=2$, $C=2$ or $A=5, B=2, C=1$ (abbreviated as the $5: 2: 2$ and $5: 2: 1$ case, respectively) the flow has no stagnation points. Numerical simulations were performed for both cases and the results obtained are similar. Therefore, only the former case is discussed in detail.

The $5: 2: 2$ flow has four points where the velocity is close to zero (but not zero, hence there are no stagnation points). The disappearance of the stagnation points means that there are no longer any heteroclinic stream lines that connect the points of minimum velocity. However, there are stream lines that converge to these points and stream lines that diverge from these points (Fig. 9). The stream lines of the $5: 2: 2$ flow do not follow any specific symmetry as in the normal $A B C$ flow (which has a three-fold symmetry of stream lines around the stagnation points) and the topology of the flow becomes more complex. 


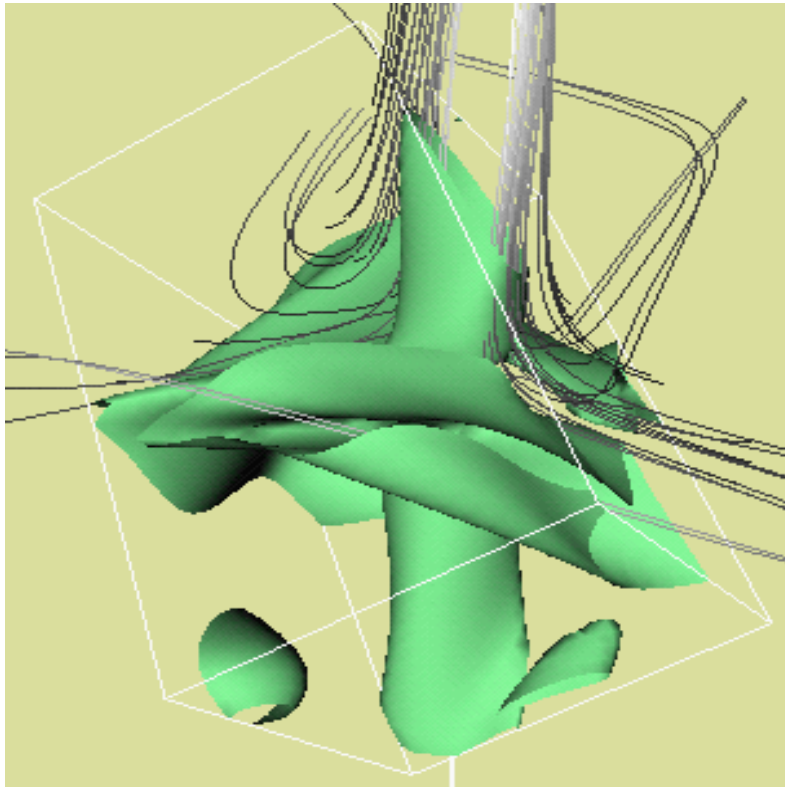

Fig. 8. Folding of week flux sheets at the plane of a $\beta$-type stagnation point. The field lines (top of the figure) are coming from a neighboring flux cigar and change direction across the plane.

The importance of these minimum velocity points is that they coincide with local extrema of the "perpendicular convergence" of the local motion to a magnetic field line

$D_{\perp} \ln \boldsymbol{B} / D t=-\nabla \cdot \boldsymbol{u}_{\perp}$

which, apart from diffusive effects, gives the exponential rate of increase of the local magnetic field amplitude (Nordlund et al. 1994). In general, it is the gradients of the flow velocity that enable the stretching of field lines that is so essential to the dynamo mechanism, and consequently these points are points where the stretching is at maximum.

\subsection{Growth rate}

Numerical experiments were performed with $R e_{\mathrm{m}}$ up to 3200 and numerical resolution up to $240^{3}$ to test whether the 5 : $2: 2$ case has vigorous dynamo properties in the kinematic regime. The initial seed field was a random perturbation with an amplitude of $10^{-5}$. It was found that the magnetic field is amplified exponentially and the growth rate increases with $R e_{\mathrm{m}}$ as illustrated in Fig. 10.

The $5: 2$ : 1 case was also examined at high magnetic Reynolds number. The highest value of $R e_{\mathrm{m}}$ considered here is $R e_{\mathrm{m}}=3200$. The growth rate seems to saturate at a value of about 0.67 as illustrated in Fig. 11.

\subsection{Flux ribbons}

As mentioned above, the $5: 2: 2$ flow has four points with minimum velocity that coincide with local maxima of the function in Eq. (4).

When the induction equation is evolved from a weak random field, flux ribbons arise close to the minimum velocity points. The thickness of the ribbons decreases as $R e_{\mathrm{m}}$ increases,

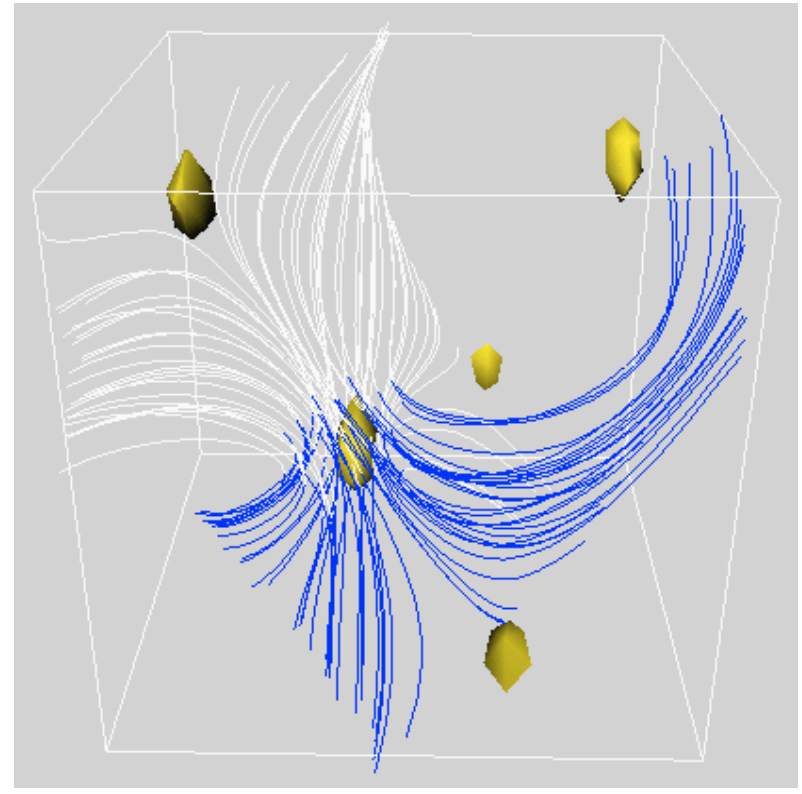

Fig. 9. The bead-shaped isosurfaces show the position of the points with minimum velocity of the $5: 2: 2$ flow. Visualization of stream lines of the flow shows that they diverge when they reach the neighborhood of other points with minimum velocity.

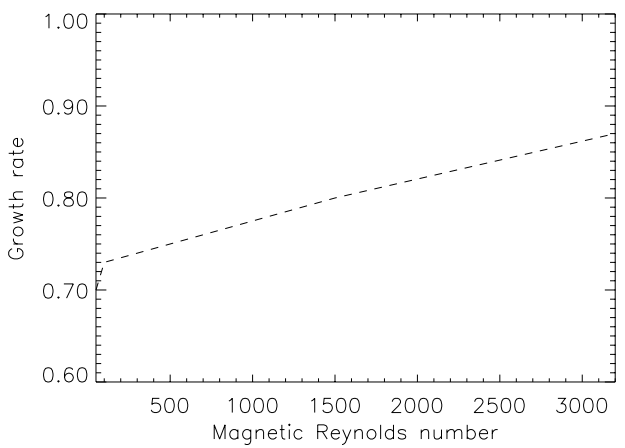

Fig. 10. Growth rate of the magnetic energy versus the magnetic Reynolds number for the $5: 2: 2$ flow.

presumably scaling as $R e_{\mathrm{m}}^{-1 / 2}$, similarly to the flux cigars for the standard $A B C$ flow. The sign (polarity) of their magnetic field changes from ribbon to ribbon. Thus, field lines with opposite polarity may reconnect when two ribbons are close together. The magnetic flux is released through the reconnected field lines (Fig. 12). However, the magnetic ribbons are not confined at certain points but are advected by the flow, and field lines with the same polarity could also be found to approach each other.

\subsection{The amplification mechanism}

The process responsible for driving the amplification mechanism of the mode with the largest growth is the constructive folding of the ribbons.

Two of the most important properties of the $5: 2: 2$ flow is the exponential stretching of the field lines and the way in which the magnetic field is advected and the field lines are packed. Neighboring regions with magnetic field of opposite 


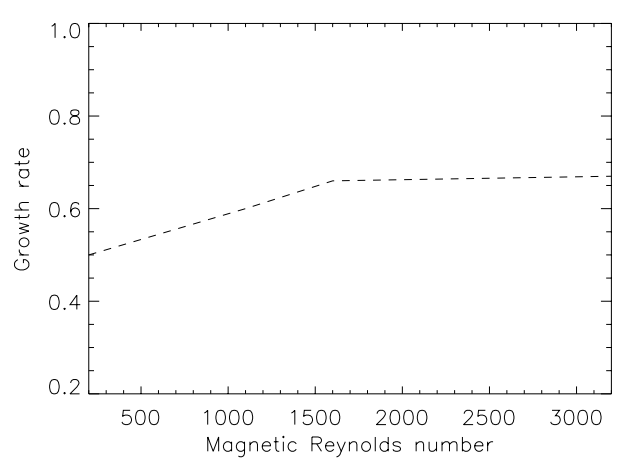

Fig. 11. Growth rate of the magnetic energy versus the magnetic Reynolds number for the $5: 2: 1$ flow.

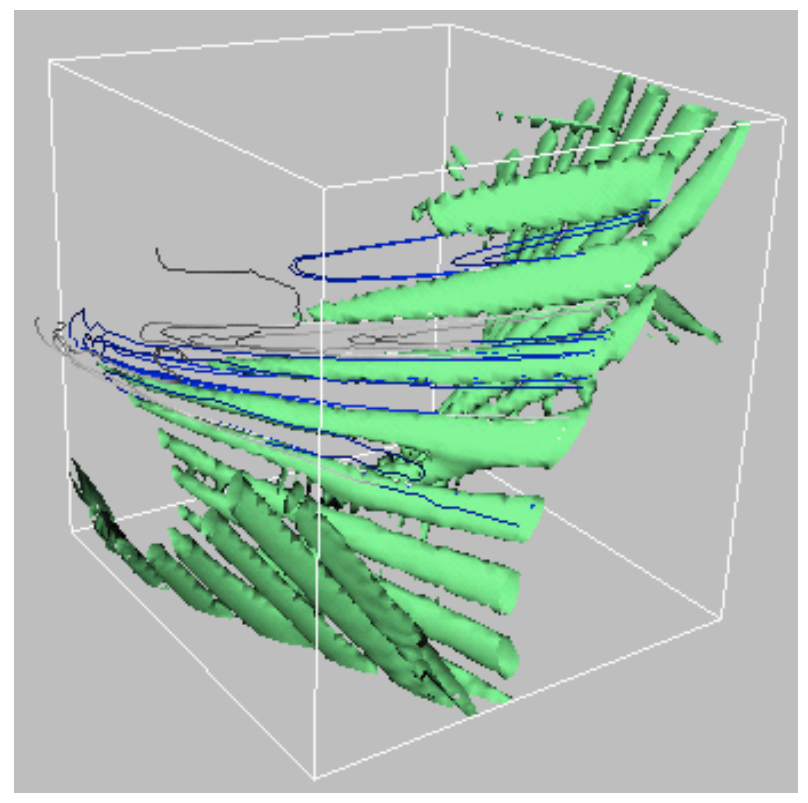

Fig. 12. The magnetic field takes the form of flux ribbons when $A: B$ : $C=5: 2: 2$. The reconnection of field lines is shown when ribbons with opposite polarity field are found close together.

polarity are destroyed whereas regions with like polarity flux are conserved. Therefore the rate of field generation depends on the global folding of the field lines.

The flow folds the field so as to bring together flux in the same direction. Thus, stretched flux ribbons with the same polarity come into alignment and merge. The constructive folding of the sheets occurs in the vicinity of the minimum velocity points (Fig. 13). Due to the stretching ability of the flow, which is maximum close to these points, the field lines are stretched in these regions.

The flow advects the field so as to bring together stretched field lines with the same polarity. They come into alignment and are folded constructively. These field lines are continuously stretched and also advected by the flow, and move to the vicinity of another neighboring point with minimum velocity, where they fold with new stretched field lines of the same polarity. The folding is again constructive.

Due to the stretching and constructive reinforcement of the magnetic field discussed above, the magnetic energy increases each time the process is repeated.

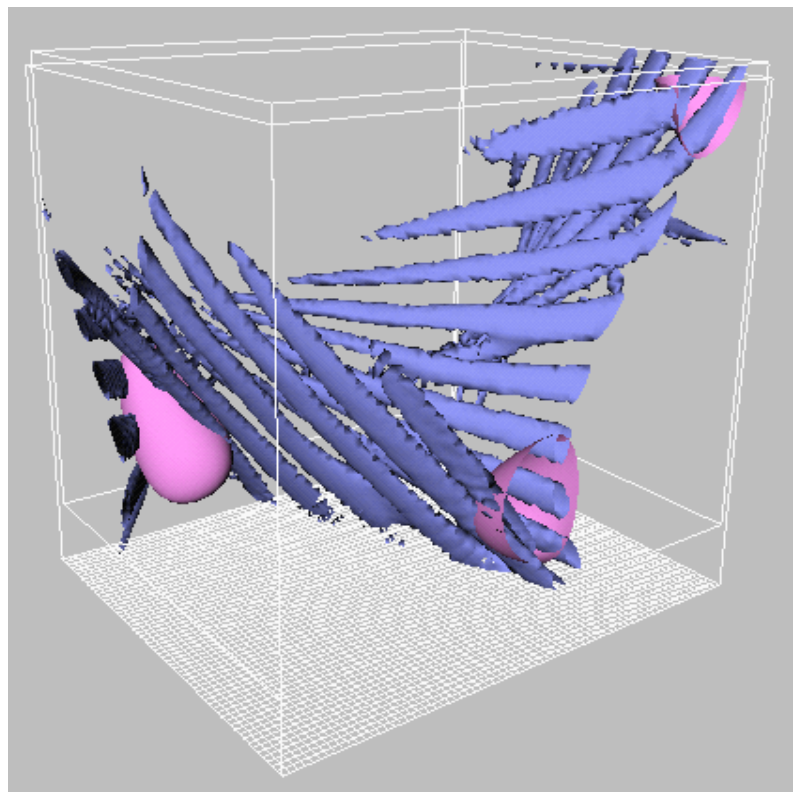

Fig. 13. A snapshot showing the merge of the ribbons (dark) with like polarity in the neighborhood of the minimum velocity points (light).

\section{Conclusions}

The amplification of the dynamo-generated field is associated with a combination of stretching, twisting, folding and reconnection of magnetic field lines. The importance of the weak part of the field in the dynamo process is emphasized when the wavenumber of the flow increases. Symmetry breaking allows the transportation of stretched weak magnetic field from cell to cell, where it gets packed against the local flux structures ("cigars"). Most of the space in the bulk part of the flow has passive advection, which does not change at increasing magnetic Reynolds number. The evidence indicates that the invariant properties of the flows (i.e. stretching ability) do not change in the limit of arbitrarily small magnetic diffusivity and the growth rate of the normal $A B C$ flow reaches an asymptotic value (for high $R e_{\mathrm{m}}$ ) even after the onset of its hydrodynamic instability.

The study of kinematic dynamo action in $A B C$ flows is, from a physical point of view, simple enough since it does not contain the complexity of non-linear back-reaction of the magnetic field. A fully non-linear study of the dynamo problem connects the initial phase of the growth of a weak magnetization state with the saturation of the dynamo generated field.

We have demonstrated that the fast dynamo action reflects the same mechanisms at work whatever the values of the parameters (such as $R e_{\mathrm{m}}$ and $k$ ) are. The main mechanism is the stretching of the weak magnetic field. This is a first step towards the understanding of the processes at work in a turbulent environment and during the saturation state of a dynamo where the flow has still a good grip on the weakest part of the magnetic field.

It is of course also of great interest to perform dynamo simulations in more realistic (e.g. spherical) geometries, and to include more physical processes such as differential rotation and 
convection. The advantage of the simulations presented in the current paper is that the individual mechanisms are easily identified and can be studied in great detail. Thus, kinematic dynamo experiments such as these can provide very useful clues for the general theory of astrophysical dynamos.

Acknowledgements. VA thanks the EU-TMR for support through a Marie Curie Fellowship. SBFD was supported through an EU-TMR grant to the European Solar Magnetometry Network. The work of ÅN was supported in part by the Danish National Research Foundation, through its establishment of the Theoretical Astrophysics Center. Computing time at the UNI•C computing center was provided by the Danish Natural Science Research Council.

\section{References}

Arnold, V., \& Korkina, E. 1983, Vest. Mosk. Un. Ta. Ser. 1, Matem. Mekh., 3, 43

Cattaneo, F., Hughes, D. W., \& Kim, E. 1996, Phys. Rev. Lett., 76, 2057
Childress, S., \& Gilbert, A. D. 1995, Stretch, Twist, Fold: The Fast Dynamo (Springer-Verlag, Berlin), 52

Dombre, T., Frisch, U., Green, J., et al. 1986, JFM, 167, 353

Dorch, S. B. F. 2000, Phys. Scr., 61, 717

Galanti, B., Sulem, P. L., \& Pouquet, A. 1992, Geophys. \& Astroph. Fluid Dyn., 66, 183-208

Galloway, D., \& Frisch, U. 1984, Geophys. \& Astroph. Fluid Dyn., 29,13

Galloway, D., \& Frisch, U. 1986, Geophys. \& Astroph. Fluid Dyn., 36,53

Galsgaard, K., \& Nordlund, Å. 1997, J. Geophys. Res., 102, 219

Hyman, J. M. 1979, Adv. Comp. Meth. for PDEs-III, ed. R. Vichnevetsky, \& R. S. Stepleman, Publ. IMACS, 313

Lau, Y.-T., \& Finn, J. M. 1993, Phys. Fluids B, 5, 365

Nordlund, A., Brandenburg, A., Jennings, R. L., et al. 1992, ApJ, 392, 647

Nordlund, A., Galsgaard, K., \& Stein, R. F. 1994, in Solar Surface Magnetic Fields, ed. R. J. Rutten, \& C. J. Schrijver, NATO ASI Series, 433

Vainshtein, S. I., \& Zeldovich, Ya. B. 1972, Usp. Fiz. Nauk, 106, 431

Podvigina, O., \& Pouquet, A. 1994, Physica D, 75, 475-508 